\title{
Influence of Glucose and Dissolved Oxygen Concentrations on Yields of Escherichia coli B in Dialysis Culture
}

\author{
By PETER LANDWALL AND TORD HOLME \\ Department of Bacteriology, Karolinska Institutet, S-104 or Stockholm, Sweden
}

(Received 3I May 1977)

\begin{abstract}
Yields of Escherichia coli B grown on glucose were determined in dialysis and non-dialysis culture. The molar growth yields were compared under conditions of excess glucose and oxygen as well as glucose- and oxygen-limiting conditions. The molar growth yields on glucose $\left(Y_{G}\right)$ were determined for different periods during growth in non-dialysis cultures. A rapid decrease of $Y_{G}$ was observed and growth ceased even in the presence of high concentrations of glucose and dissolved oxygen in the culture liquid. The decrease in $Y_{\mathrm{G}}$ was delayed in dialysis cultures where a high $Y_{G}$ could be maintained at very high cell concentrations. The inhibition of growth depended on the accumulation of end-products of fermentative degradation of glucose. These products interfered with the oxidative phosphorylation. A large proportion of the glucose was fermented even in the presence of high concentrations of dissolved oxygen in the culture liquid. A decrease in the growth yield per $\mathrm{g}$ glucose was also observed.
\end{abstract}

\section{INTRODUCTION}

We have previously shown, by the use of dialysis culture, that growth-inhibitory metabolites accumulate in the culture fluid of cultures of Escherichia coli в (Landwall \& Holme, 1977). We also observed that the molar growth yield on glucose ( $\mathrm{g}$ bacterial dry wt per mol glucose consumed, $Y_{\mathrm{a}}$ ) varied during growth. In cultures where the dissolved oxygen concentration was kept above $20 \%$ of saturation, $Y_{G}$ was initially between 85 and 100. During the cultivation, $Y_{\mathrm{G}}$ decreased very rapidly in non-dialysis culture, but in dialysis culture this decrease occurred much later, indicating that the metabolites removed by dialysis exerted their inhibitory action by interfering with the energy metabolism of the bacteria.

The important factors influencing growth yields of facultative bacteria in minimal medium are the concentrations of glucose and dissolved oxygen. The feeding of nutrients to the growing culture permitted the maintenance of glucose concentrations exceeding Io $\mathrm{g}^{-1}$ during the whole culture period, as well as glucose-limiting conditions.

In the present paper we report studies of the influence of the concentrations of glucose and dissolved oxygen on molar growth yields in dialysis and non-dialysis culture. The oxygen consumption of the bacteria was measured, and the growth yield on glucose oxidized for different periods of the cultivation could then be calculated. The results indicate that end-products of the fermentative utilization of glucose interfered with the energy metabolism by inhibiting oxidative phosphorylation as well as making it less effective with respect to growth yield.

\section{METHODS}

Bacterial strain. Escherichia coli strain B was used in these studies.

Cultivation. The culture equipment and conditions were as described previously (Landwall \& Holme, 1977). Oxygen consumption was measured by analysing the $\mathrm{O}_{2}$ concentration in the outgoing gas from the fermen- 
ter, using a paramagnetic analyser (Magnos 2, Hartmann \& Braun, Frankfurt/Main, Germany) connected to a recorder. Glucose was determined by the glucose oxidase method (Glox, Kabi, Stockholm, Sweden). End-products formed by the culture were analysed by gas chromatography as described previously (Landwall \& Holme, 1977). The salt concentrations were estimated by conductivity measurements (conductivity meter $\mathrm{CDM}_{3}$, Radiometer, Copenhagen, Denmark). Glucose-limited growth with oxygen in excess was obtained by feeding glucose at a constant rate to the growing culture, as described previously (Landwall \& Holme, 1977; Pirt, 1975). Oxygen was kept in excess by mixing the ingoing air with oxygen by manual control. The dissolved oxygen tension was kept at values above $20 \%$ of saturation. In oxygen-limited cultures, the air input was adjusted to give an oxygen transfer rate just below that required for the expected oxidation of the glucose. All results were confirmed by repeating each culture once or twice.

Calculations. In the culture systems used, four sets of conditions were maintained and compared in dialysis and non-dialysis culture: (i) glucose-limited with oxygen in excess; (ii) oxygen-limited with glucose in excess; (iii) limited by both oxygen and glucose; and (iv) with both oxygen and glucose in excess. From measurements of the oxygen concentration in the outgoing gas the oxygen consumption of the culture was determined and the amount of glucose oxidized per $\mathrm{g}$ dry wt bacteria formed was calculated. Knowing the total amount of glucose consumed and correcting for the glucose carbon assimilated, the amount of glucose fermented could be calculated. As a control for this calculation, the amount of organic acids formed during cultivation was roughly determined from the amount of alkali needed for pH-control, assuming 2.5 acid equivalents formed per mol glucose fermented. Correction was made for ammonium ions consumed. Endproduct analysis by gas-liquid chromatography also confirmed that the quantities of organic acids were in the range expected from the calculations.

\section{RESULTS}

Results from four non-dialysis cultures, grown under different conditions with respect to glucose concentration and dissolved oxygen, are shown in Fig. I. The $Y_{\mathrm{a}}$ values were calculated for each hour of the cultivation and the values for each 2 or $3 \mathrm{~h}$ period were plotted against the bacterial dry weight yields. With oxygen in excess, the growth curves were similar in the cultures with and without glucose limitation (Fig. I $c$ ), but the glucose consumption was much higher in the culture with glucose in excess (Fig. $1 a$ ). The overall $Y_{\mathrm{a}}$ values at a bacterial density of $34 \mathrm{~g}^{-1}$ were 44 and $32 \mathrm{~g}$ per mol glucose for the cultures with and without glucose limitation, respectively. In the oxygen-limited cultures, the final bacterial yields were approximately half of those obtained with oxygen in excess (Fig. I $d$ ) and $Y_{\mathrm{a}}$ decreased very rapidly with increasing cell densities (Fig. I $b$ ). The growth curves in the two oxygen-limited cultures were similar (Fig. I $d$ ).

The $Y_{G}$ values in the dialysis cultures are plotted against the bacterial dry weight yields in Fig. 2. Comparison with cultures grown under the same conditions except for dialysis (Fig. I) indicates that the $Y_{G}$ values for each individual point are higher in dialysis culture. The lower $Y_{\mathrm{G}}$ values in non-dialysis culture may be taken as a measure of the degree of inhibition caused by diffusible metabolites.

The ratios of the bacterial dry weight yields at $Y_{G}=60 \mathrm{~g} \mathrm{~mol}^{-1}$ in dialysis cultures to those in non-dialysis cultures under the different cultivation conditions are given in Table $I$. The largest difference in cell yields between dialysis and non-dialysis cultures was found in glucose-limited cultures with oxygen in excess. When glucose was kept in excess (10 to 20 $\mathrm{g}^{-1}$ ) by feeding it to the growing cultures, cell yields were much lower. The differences in cell yields between dialysis and non-dialysis cultures were most marked when oxygen was in excess.

By measuring oxygen consumption, it was possible to estimate the amount of glucose oxidized at different parts of the growth curve and under the different conditions applied. The growth yields were calculated for each hour during the cultivation. In Fig. 3, the amounts of glucose oxidized and glucose fermented $\mathrm{h}^{-1}$ ( $\mathrm{g}$ dry wt bacteria formed $)^{-1}$ are shown at the four points on each growth curve where the $Y_{\mathrm{O}_{2}}$ ( $\mathrm{g}$ bacterial dry wt per mol oxygen consumed) values were $60,40,20$ and I0. In a glucose-limited culture with oxygen in excess (Fig. $3 a$ ), fermentation was repressed up to very high cell densities in the dialysis culture. The amount of glucose oxidized increased during the last period of growth with a simul- 

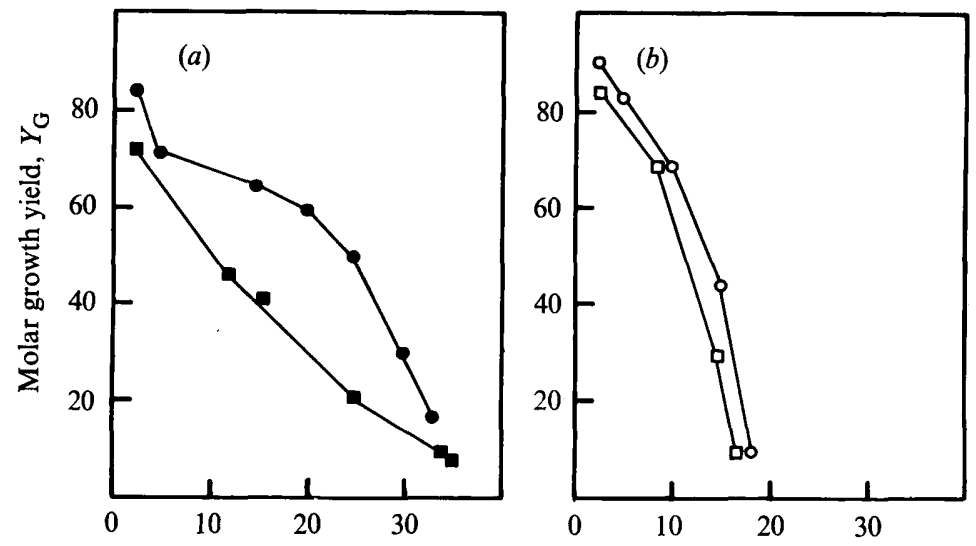

Bacterial dry weight $\left(\mathrm{g}^{-1}\right)$

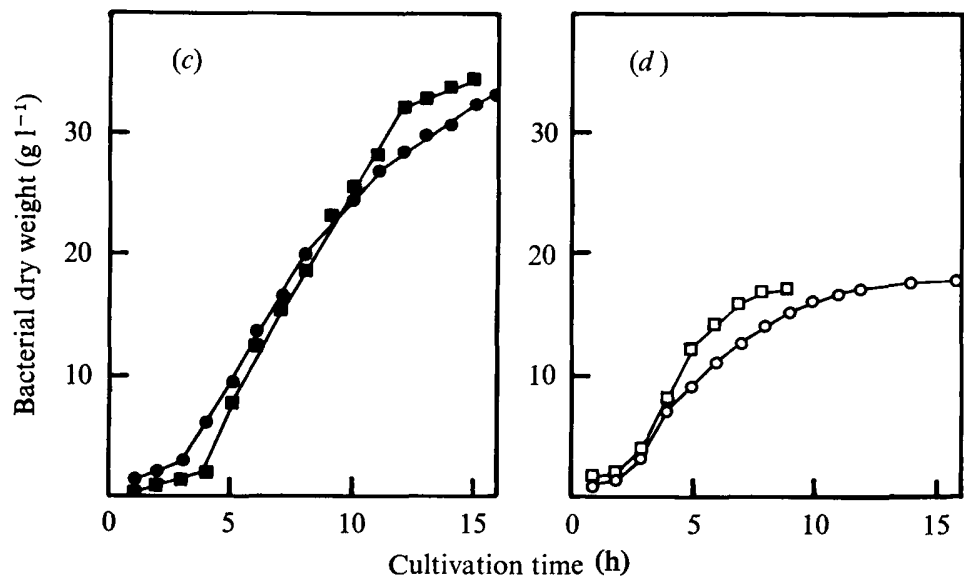

Fig. I. $Y_{a}$ values calculated for regular time intervals in non-dialysis cultures growing under different conditions $(a, b)$. Corresponding growth curves are also represented $(c, d)$. $\bigcirc$, Glucoselimited, oxygen in excess ( $>20 \%$ of saturation); $\square$, glucose in excess $\left(>5 \mathrm{~g} \mathrm{l}^{-1}\right.$ ), oxygen in excess ( $>20 \%$ of saturation); $O$, glucose-limited, oxygen-limited; $\square$, glucose in excess $\left(>5 \mathbf{g ~ 1}^{-1}\right)$, oxygenlimited.

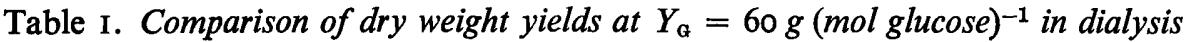
and non-dialysis cultures

\begin{tabular}{|c|c|c|c|}
\hline \multirow[b]{2}{*}{$\begin{array}{l}\text { Cultivation } \\
\text { conditions* }\end{array}$} & \multicolumn{2}{|c|}{ Bacterial dry weight $\left(\mathrm{g} \mathrm{l}^{-1}\right)$} & \multirow{2}{*}{$\begin{array}{l}\text { Ratio of dry weight } \\
\text { yields (dialysis/non- } \\
\text { dialysis culture) }\end{array}$} \\
\hline & $\begin{array}{l}\text { Dialysis } \\
\text { culture }\end{array}$ & $\begin{array}{l}\text { Non-dialysis } \\
\text { culture }\end{array}$ & \\
\hline $\begin{array}{l}\text { DO > } 20 \% \\
\text { Glc-limited }\end{array}$ & 130 & 15 & $8 \cdot 7$ \\
\hline $\begin{array}{l}\text { DO }>20 \% \\
\text { Glc }>5 \mathrm{~g} \mathrm{I}^{-1}\end{array}$ & 29 & 5 & $5 \cdot 8$ \\
\hline $\begin{array}{l}\text { DO } \rightarrow 0 \\
\text { Glc-limited }\end{array}$ & 28 & I I & $2 \cdot 5$ \\
\hline $\begin{array}{l}\text { DO } \rightarrow 0 \\
\text { Glc }>5 \mathrm{~g} \mathrm{l}^{-1}\end{array}$ & 10 & 9 & $\mathbf{I} \cdot \mathbf{I}$ \\
\hline
\end{tabular}

* Glc, glucose; DO, dissolved oxygen ( $\%$ of saturation); DO $\rightarrow 0$, dissolved oxygen was initially $100 \%$, but decreased during cultivation and became zero at a cell density of $6 \mathrm{~g} \mathrm{l}^{-1}$; further growth was thus oxygenlimited. 


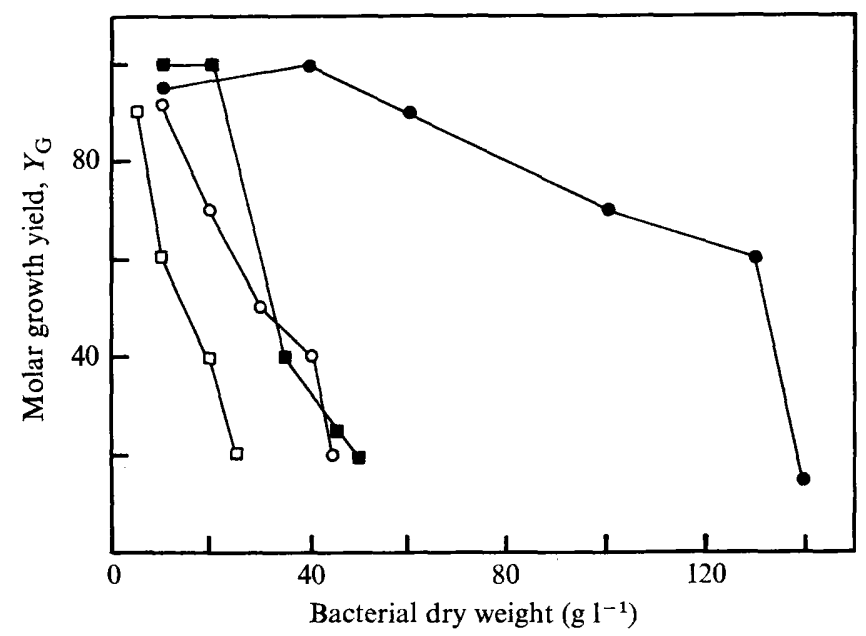

Fig. 2. $Y_{G}$ values calculated for different time intervals in dialysis cultures growing under different conditions. Glucose-limited, oxygen in excess ( $>20 \%$ of saturation); $\square$, glucose in jexcess ( $>5 \mathrm{~g}^{-1}$ ), oxygen in excess ( $>20 \%$ of saturation); $\bigcirc$, glucose-limited, oxygen-limited; $\square$, glucose in excess $\left(>5 \mathrm{~g} \mathrm{1}^{-1}\right)$, oxygen-limited.

taneous steep rise in the amount of glucose fermented. In the corresponding non-dialysis culture (Fig. $3 a$ ), the amount of glucose fermented was much higher at low cell densities and already exceeded the glucose oxidized at a bacterial concentration of $\mathrm{I} 2 \mathrm{~g}$ dry wt $\mathrm{1}^{-1}$, even though the partial pressure of dissolved oxygen was kept at values above $20 \%$ of saturation. With glucose in excess (Fig. $3 d$ ), the amount of glucose fermented increased in both the dialysis and the non-dialysis culture. In the culture limited both by oxygen and glucose (Fig. $3 c$ ), fermentation was repressed very effectively in the dialysis culture. In oxygen-limited cultures with glucose in excess (Fig. $3 b$ ), the difference between dialysis and non-dialysis cultures was not so marked as in the other experiments, but the same early repression of fermentation was noted as well as an increased efficiency of glucose utilization in dialysis culture.

As shown earlier (Landwall \& Holme, 1977), growth inhibition was caused by the combined action of several end-products of fermentative degradation of glucose. Acetate alone was not growth-inhibitory at $\mathrm{pH} 7 \cdot 0$ in concentrations of $20 \mathrm{~g} \mathrm{l}^{-1}$, but growth inhibition in both dialysis and non-dialysis culture occurred when the acetate had accumulated to between 7 and $13 \mathrm{~g} \mathrm{1}^{-1}$. Acetate concentration was therefore chosen as a measure of the amount of organic acids accumulated. The other acids accumulated in proportional amounts (lactic acid, I to $2 \mathrm{~g}^{-1}$; pyruvic acid, I to $2 \mathrm{~g}^{-1}$; succinic acid, I to $2 \mathrm{~g}^{-1}$; propionic acid, 0.2 to $0.5 \mathrm{~g}^{-1}$; and isobutyric acid, 0.2 to $0.5 \mathrm{~g} \mathrm{l}^{-1}$ ). In non-dialysis cultures the bacterial density at which growth-inhibitory concentrations of these metabolites were reached depended on the glucose and dissolved oxygen concentrations (Fig. 3). In dialysis culture, the inhibitory concentrations were reached at a much higher bacterial density because of the continuous diffusion of the metabolites from the culture to the reservoir (Fig. 3).

In the dialysis culture growing under glucose-limited conditions with oxygen in excess, the acetate concentration in the culture fluid was $4.5 \mathrm{~g}^{-1}$ at a bacterial density of $100 \mathrm{~g}^{1^{-1}}$, but increased to $8.5 \mathrm{~g} \mathrm{1}^{-1}$ at a bacterial dry weight of $130 \mathrm{~g}^{-1}$. Under other cultivation conditions in dialysis culture, accumulation of metabolites in the culture fluid was also found at the time when growth inhibition was observed. In the culture with both oxygen and glucose in excess, the final concentration of acetate was $8.5 \mathrm{~g}^{-1}$ (bacterial density 47 $\mathrm{g}^{-1}$ ) and in the oxygen-limited culture with glucose in excess, the acetate concentration was $9 \mathrm{~g}^{-1}$ at the end of growth (bacterial density $22 \mathrm{~g}^{-1}$ ). 


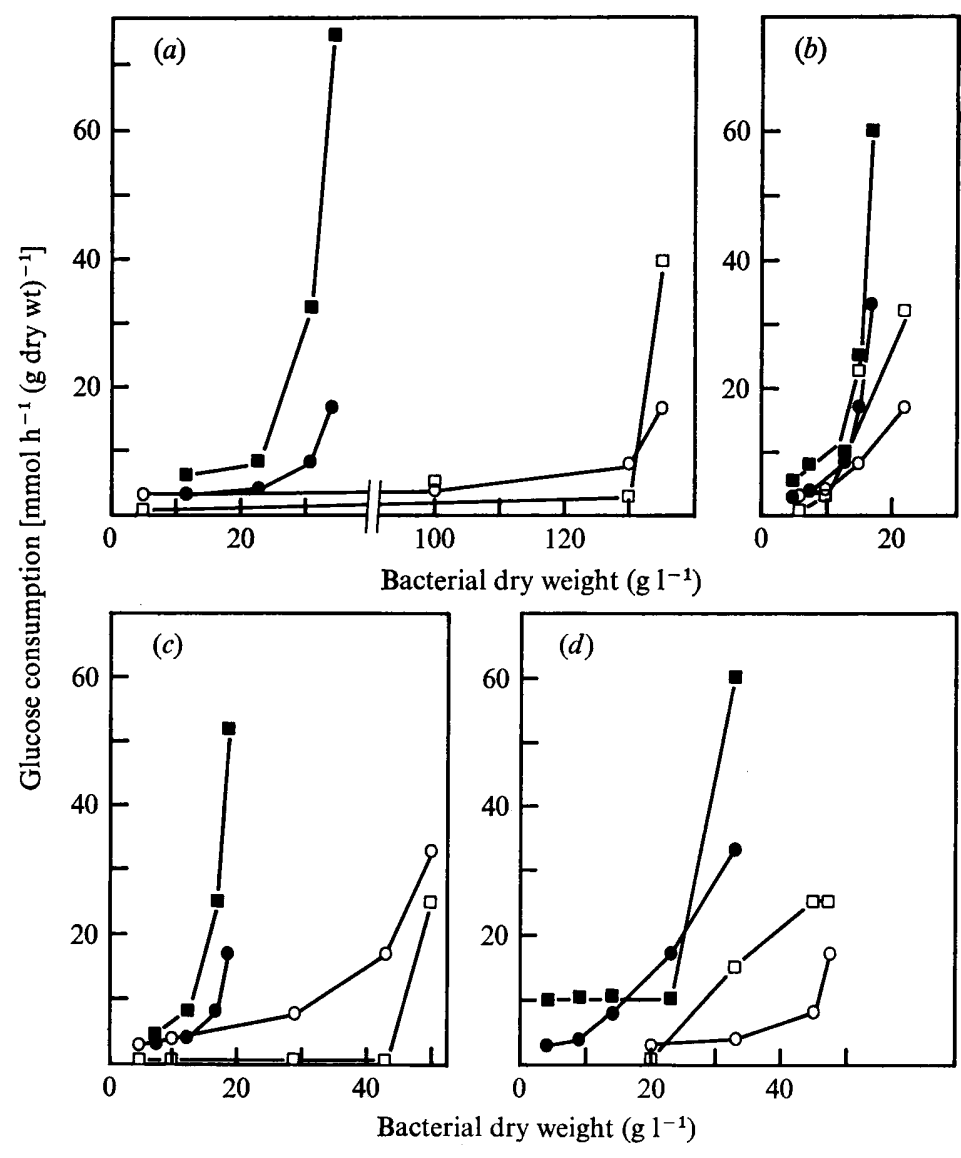

Fig. 3. Glucose consumption $\left[\mathrm{mmol} \mathrm{h}^{-1}\right.$ (g dry wt bacteria formed)-1] by fermentation $(\square, \square)$ and oxidation $(O, \Theta)$ in dialysis $(\square, O)$ and non-dialysis $(\square, O)$ cultures under different conditions. The points show the values obtained at successive $Y_{\mathrm{O}_{2}}$ values of $60,40,20$ and 10 . (a) Glucose-limited, oxygen in excess ( $>20 \%$ of saturation); (b) glucose in excess $\left(>5 \mathrm{gl}^{-1}\right.$ ), oxygenlimited; $(c)$ glucose-limited, oxygen-limited; $(d)$ glucose in excess $\left(>5 \mathrm{~g} \mathrm{l}^{-1}\right)$, oxygen in excess (>20\% of saturation).

The concentrations of the metabolites in the reservoir were also determined. At the end of the cultivations, the acetic acid concentration in the reservoir was approximately 6 to 7 $\mathrm{g} \mathrm{l}^{-1}$, showing that the limitation of dialysis capacity depended more on the reservoir volume than the membrane area.

\section{DISCUSSION}

Previously, we demonstrated that the removal of extracellular, dialysable metabolites increased the bacterial yields of cultures of $E$. coli B growing in a glucose/salts medium (Landwall \& Holme, 1977). The inhibitory metabolites which accumulated in the cultures were identified as end-products of glucose fermentation. In combination, they were shown to decrease the growth yield on glucose, $Y_{\mathrm{G}}$. Since aeration conditions and glucose concentrations during growth have a great influence on the formation of end-products of glucose metabolism, the present studies were undertaken. The results indicate that the decreased yields resulted from a combination of at least two effects. First, the ratio of glucose fermented to glucose oxidized increased with the presence of the metabolites. This can be seen at the early stages of all cultures by comparing dialysis and non-dialysis cultures (Fig. 3). Second, the efficiency of substrate conversion by oxidative phosphorylation 
decreased. This is best illustrated in the dialysis culture shown in Fig. $3(c)$, where there was an increase in the amount of glucose oxidized per $g$ dry wt bacteria produced from 3 to 18 mmol during a period in which there was no increase in the amount of glucose fermented.

The increase in the amount of glucose fermented per $\mathrm{g}$ dry wt bacteria produced was also influenced by dialysis. This effect was observed very early in non-dialysis cultures, even when the aeration efficiency was high. It was also influenced by the glucose concentration. It is not possible to deduce from the present results whether the increase in fermentation was itself promoted by the concomitant accumulation of the products of fermentation. It is well established that acetate is growth-inhibitory, but only at $\mathrm{pH}$ values below 7 where there is a greater fraction present as undissociated acid. Sheu \& Freese (1972) showed that $0 \cdot 1$ to $0.2 \mathrm{M}$-acetate interfered with the electron transport system in Bacillus subtilis, but not with sugar uptake or ATP production via glycolysis. Inhibition of oxygen uptake was also shown and the results indicated that acetate (and other fatty acids) inhibited the formation of reducing compounds, which could be oxidized by the electron transport system. In contrast, no inhibition of growth of $E$. coli at $\mathrm{pH} 7 \cdot 0$ could be demonstrated in the present study when $0.2 \mathrm{M}$-sodium acetate was included in the nutrient medium, indicating that acetate was not per se a growth-inhibitory metabolite.

The present results show that dialysis culture is a very valuable method for analysing growth inhibition. Other methods for removing auto-inhibitory metabolites should be tested, but dialysis culture seems to be the most widely applicable method. Since most of the auto-inhibitory metabolites seem to decrease the substrate conversion efficiency, it must be considered to be of great importance to reduce their effects in microbial fermentations.

This work was supported by grants from the Swedish Board for Technical Development (73-3744, 74-3469) and from Emil and Wera Cornells Stiftelse. Our thanks are due to Tove Harde for skilful technical assistance.

\section{REFERENCES}

Landwall, P. \& Holme, T. (1977). Removal of inhibitors of bacterial growth by dialysis culture. Journal of General Microbiology 103, 345-352.

PIRT, S. J. (1975). Batch cultures with substrate feeds. In Principles of Microbe and Cell Cultiva- tion, p. 2I I. Oxford: Blackwell Scientific Publications.

Sheu, C. W. \& Freese, E. (1972). Effects of fatty acids on growth and envelope proteins of Bacillus subtilis. Journal of Bacteriology III, 516-524. 